\title{
High Precision Infrared Temperature Measurement System Based on Distance Compensation
}

\author{
Jing Chen ${ }^{1}$, Ji-Ping Wang ${ }^{1}$, Tian-Yu Shen ${ }^{1,2}$, Da-Xi Xiong ${ }^{1}$, and Li-Quan Guo ${ }^{1, a}$ \\ ${ }^{1}$ Suzhou Institute of Biomedical Engineering, Chinese Academy of Sciences, 215163 Suzhou, China \\ ${ }^{2}$ School of communication and information engineering, Shanghai University, 200444 Shanghai, China \\ a Corresponding author: guolq@sibet.ac.cn
}

\begin{abstract}
To meet the need of real-time remote monitoring of human body surface temperature for optical rehabilitation therapy, a non-contact high-precision real-time temperature measurement method based on distance compensation was proposed, and the system design was carried out. The microcontroller controls the infrared temperature measurement module and the laser range module to collect temperature and distance data. The compensation formula of temperature with distance wass fitted according to the least square method. Testing had been performed on different individuals to verify the accuracy of the system. The results indicate that the designed non-contact infrared temperature measurement system has a residual error of less than $0.2^{\circ} \mathrm{C}$ and the response time isless than $0.1 \mathrm{~s}$ in the range of 0 to $60 \mathrm{~cm}$. This provides a reference for developing longdistance temperature measurement equipment in optical rehabilitation therapy.
\end{abstract}

\section{Introduction}

Optical rehabilitation therapy as a noninvasive physical therapy, it and has no side effects, by using different wavelengths of light to irradiate to tissue, to cause the organization of cytochrome, mitochondria and other molecular a series of biological effects to achieve the purpose of therapy [1]. These biological effects have the effect of promoting tissue wound healing, antiinflammatory analgesic, relieving muscle tissue fatigue and promoting tissue repair and so on. Therefore, optical rehabilitation therapy is widely used in the treatment of dermatology, surgery, rehabilitation and other diseases. The thermal effect of optical rehabilitation therapy can cause angiectasis, faster blood flow, and can improve microcirculation as well as immunity, but at the same time the tissue warming caused by thermal effect will cause the patient's irradiated site burns if not well controlled. Therefore, real-time monitoring of the patient's irradiated site temperature can effectively prevent burns when physiotherapy occurs.

Real-time monitoring of the temperature of the irradiated part of the patient can be used by measuring the temperature of the human body infrared radiation. Environmental factors, distance coefficient, emissivity some other factors will affect the infrared temperature accuracy [2]. According to the law of The Total Radiation of the Stewart-Boltzmann, the radiation intensity of any object is always proportional to the actual emissivity of the object. The body's skin emissivity will have some differences according to skin's state, the race, but its range is around 0.95 . The infrared temperature measurement error caused by emissivity can be ignored [3]. In view of the influence of distance factors on infrared temperature measurement, there are many research results. There is a relatively fixed relationship between the temperature error of the infrared thermometer and the distance [4]. At present, the typical solution is to use ultrasonic distance compensation to improve the accuracy of infrared temperature measurement, but the measurement distance is limited within $10 \mathrm{~cm}$.

For optical rehabilitation therapy's $0-60 \mathrm{~cm}$ long distance non-contact temperature measurement needs, a non-contact high-precision real-time infrared temperature measurement system based on distance compensation is designed. The system uses the laser distance measuring module to collect the distance numerical in real time to compensate the temperature and eliminate the influence of the distance factor on the infrared temperature measurement result. Laser ranging is not susceptible to ambient light, temperature and other factors, thus making the distance measurement system and the final temperature monitoring very stable.

\section{System Design}

The basic principle of the non-contact temperature measurement system based on distance compensation is to measure the surface temperature by the high-precision infrared temperature measurement module and measure the distance between the detection terminal and the 
human body in real time. The distance compensation is compensated by the distance compensation formula to meet the needs of long-range non-contact temperature measurement [5].

The system block diagram shown in Figure 1. mainly includes micro-controller, high-precision infrared temperature measurement module, laser distance measuring module and display modules and other parts. Microcontroller is the high-performance ARM chip STM32F103ZET6. The main controller collects the highprecision infrared temperature measurement module MLX90614's temperature data through the SMbus in real time and synchronously acquires laser distance measurement module VL53L0X's distance data through the IIC bus. And then it analyzes and processes the data and uploads the processed temperature value to the PC for display and storage through the USB to RS232 Bridge.

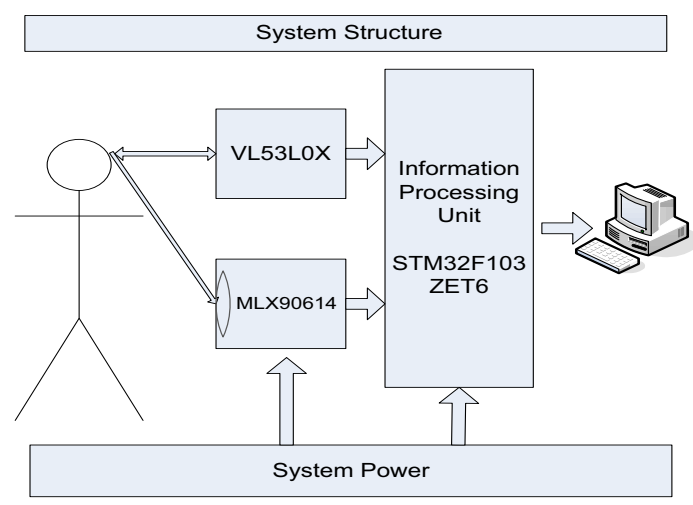

Figure 1. System Block Diagram.

The high precision infrared temperature measurement module MLX90614 internal integrates infrared thermopile sensor MLX81101 and signal processing dedicated integrated chip MLX90302. The MLX90302 is designed to handle the output signals of infrared sensor. MLX90302 measures the object and ambient temperature by the internal state machine, and then calculates and outputs the calculation results through the SMbus. In the range of $0-120^{\circ} \mathrm{C}$, MLX90614 measurement accuracy can reach $0.5^{\circ} \mathrm{C}$, for the body temperature measurement of about $37^{\circ} \mathrm{C}$, the measurement accuracy can be up to about $0.1^{\circ} \mathrm{C}$.

The MLX90614 module also integrates filters to block visible and near-infrared radiation, and the wavelength passband of the filter is $5500 \mathrm{~nm}$ to $14000 \mathrm{~nm}$. The radiation wavelength of the human body is near $9000 \mathrm{~nm}$, and the optical therapy exposure wavelength range is $200 \mathrm{~nm}$ to $1400 \mathrm{~nm}$. So the filter can effectively filter out the impact of ambient light and light of physical therapy.

The optical lens and the outer package determine the infrared field measuring range and the distance coefficient. Above the infrared sensor there is an eyepiece which can guarantee that a sphere with the diameter of $20 \mathrm{~cm}$ can fully-fill the entire perspective in the range of $0-60 \mathrm{~cm}$. The angle of the surrounding environment on the lens is very small, so the impact of direct thermal radiation in the environment can be ignored.
The laser distance measuring module consists of VL53L0X laser temperature sensor. The VL53L0X based on FlightSense ${ }^{\mathrm{TM}}$ technology is the world's smallest ToF (Time-of-Flight) module, it integrates light sources, SPAD photon detectors and advanced microcontrollers (managing the entire ranging function). The laser ranging module VL53L0X connects with STM32F103ZET6 through the IIC bus to read and write data.

\section{Experiment and Data Processing}

\subsection{Experiment and Data Collection}

In order to verify the performance of the system and the accuracy of temperature measurement, experiments and tests were carried out on 4 healthy adult yellow skin men(A, B, C, D)from the laboratory.

To measure the actual temperature of the irradiated area of the patient, Microlife's contact infrared temperature sensor, Model: FR1DM1, measurement accuracy $0.1^{\circ} \mathrm{C}$, was used to test the temperature of the forehead (regarded as a spherical heating element with the diameter of $20 \mathrm{~cm}$ ) as the reference value of the actual temperature of the irradiated area.

The temperature of four subjects was measured using a non-compensated infrared temperature measurement system and Microlife FR1DM1 at room temperature. The distance between 0 and $60 \mathrm{~cm}$ was divided into 6 parts. Temperature was measured three times at each point and was averaged to reduce the chance of accidental errors.

Arbitrarily selected one of the subjects' data to use the compensation algorithm to compensate for the fitting formula, and combine it to the infrared temperature measurement system. After the distance compensation, a test was carried out under the same condition to test the temperature of the four subjects to verify the distance compensation effect.

To verify the effect of different colors had on temperature measurement of the infrared temperature measuring system with distance compensation, a black and white skin temperature experiments under the same experimental conditions was carried out.

\subsection{Data Processing}

As could be seen from 4 sets of data measured by noncompensated infrared temperature measurement system, data were in line with the following trends: In the range of 0 to $20 \mathrm{~cm}$, the error was kept within $0.2^{\circ} \mathrm{C}$. But when the distance was greater than $20 \mathrm{~cm}$, the measurement error increased rapidly as the distance increases. When the distance was $60 \mathrm{~cm}$, the error reached $2^{\circ} \mathrm{C}$. Figure 2 was the relationship between the measured values $\mathrm{T}(\mathrm{O})$, $\mathrm{T}(\mathrm{A})$ of the randomly selected subjects $\mathrm{C}$ and the measured distance. 


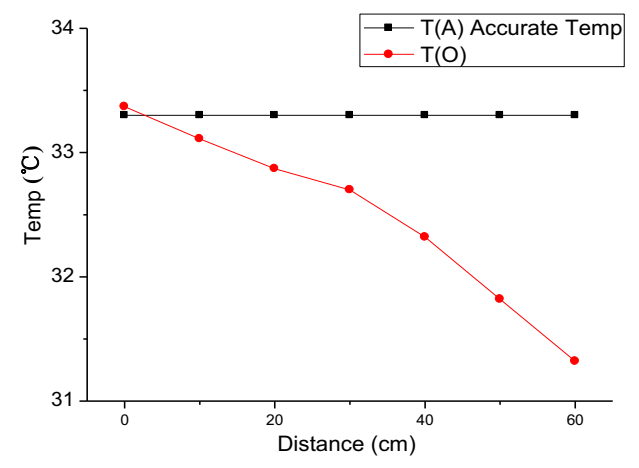

Figure 2. $\mathrm{T}(\mathrm{O}), \mathrm{T}(\mathrm{A})$ of subject $\mathrm{C}$

As the distance increased, the error between the measured value $\mathrm{T}(\mathrm{O})$ measured by the infrared temperature measurement system and the actual temperature reference value T(A) measured by Microlife FR1DM1 became larger. In order to filter the effect of the actual temperature had on the fitting results, the ratio of the value measured by non-compensated infrared temperature measurement system to the actual temperature measured by Micro life FR1DM1 was used as the measuring factor $\mathrm{Y}$ to discuss the effect of the distance ${ }^{x}$ had on temperature. The system used the least squares method to obtain the ratio of $\mathrm{T}(\mathrm{O})$ to $\mathrm{T}(\mathrm{A})$, that is, the relationship between the measured factor $\mathrm{Y}$ and the distance $^{x}$. The relationship between the measurement factor $\mathrm{Y}$ and the distance ${ }^{x}$ between $0-60 \mathrm{~cm}$ could be characterized by a quadratic function, regarded the quadratic function (1):

$Y=\hat{a}_{0}+\hat{a}_{1} x+\hat{a}_{2} x^{2}$

as the approximate fitting curve, the mean square error was as following:

$Q\left(a_{0}, a_{1}, a_{2}\right)=\sum_{i=1}^{n}\left(a_{0}+a_{1} x_{i}+a_{2} x_{i}^{2}-y_{i}\right)^{2}$

Let the mean square error (2) be the smallest, and the equation (3) was obtained by the method of finding the extreme value.

$$
\left(\begin{array}{ccc}
n & \sum_{i=1}^{n} x_{i} & \sum_{i=1}^{n} x_{i}^{2} \\
\sum_{i=1}^{n} x_{i} & \sum_{i=1}^{n} x_{i}^{2} & \sum_{i=1}^{n} x_{i}^{3} \\
\sum_{i=1}^{n} x_{i}^{2} & \sum_{i=1}^{n} x_{i}^{3} & \sum_{i=1}^{n} x_{i}^{4}
\end{array}\right)\left(\begin{array}{l}
a_{0} \\
a_{1} \\
a_{2}
\end{array}\right)=\left(\begin{array}{c}
\sum_{i=1}^{n} y_{i} \\
\sum_{i=1}^{n} x_{i} y_{i} \\
\sum_{i=1}^{n} x_{i}^{2} y_{i}
\end{array}\right)
$$

Solve $\hat{a}_{0}, \hat{a}_{1}, \hat{a}_{2}$ to get the fit formula. From the definition of the measurement factor $\mathrm{Y}$, it could be seen that the temperature after compensation $\mathrm{T}(\mathrm{C})$ is $\mathrm{T}(\mathrm{O}) / \mathrm{Y}$. Fit the above experimental data to get the distance compensation formula: $x$ was the distance, $\mathrm{Y}$ was the measurement factor, and the correlation coefficient R2 of the equation (4) was 0.9998 .

Take the fitting formula into the system for distance compensation and test the subject C's forehead temperature $\mathrm{T}(\mathrm{C})$ in the same external environment. As could be seen from Table 1, the maximum error of the system with distance compensation reduced from $2^{\circ} \mathrm{C}$ to $0.2^{\circ} \mathrm{C}$ under the same test conditions.

Table 1. Forehead temperature $\mathrm{T}(\mathrm{C})$ of subject $\mathrm{C}$.

\begin{tabular}{|c|c|c|c|}
\hline Distance $(\mathrm{cm})$ & $\mathrm{T}(\mathrm{A}){ }^{\circ} \mathrm{C}$ & $\mathrm{T}(\mathrm{O}){ }^{\circ} \mathrm{C}$ & $\mathrm{T}(\mathrm{C}){ }^{\circ} \mathrm{C}$ \\
\hline 0 & 33.3 & 33.37 & 33.43 \\
\hline 10 & 33.3 & 33.11 & 33.33 \\
\hline 20 & 33.3 & 32.87 & 33.40 \\
\hline 30 & 33.3 & 32.70 & 33.24 \\
\hline 40 & 33.3 & 32.32 & 33.34 \\
\hline 50 & 33.3 & 31.82 & 33.44 \\
\hline 60 & 33.3 & 31.32 & 33.32 \\
\hline
\end{tabular}

In order to test the effect of the fitting algorithm had on different individuals, the remaining three subjects were subjected to a temperature test with infrared temperature measurement system with distance compensation and the results were compared with Micro life FR1DM1 under the same conditions. The temperature comparison curves of the actual temperature $\mathrm{T}(\mathrm{A})$ measured by Micro life FR1DM1 and the forehead temperature $\mathrm{T}(\mathrm{C})$ measured by the infrared temperature measurement system with distance compensation were shown in Figure 3.
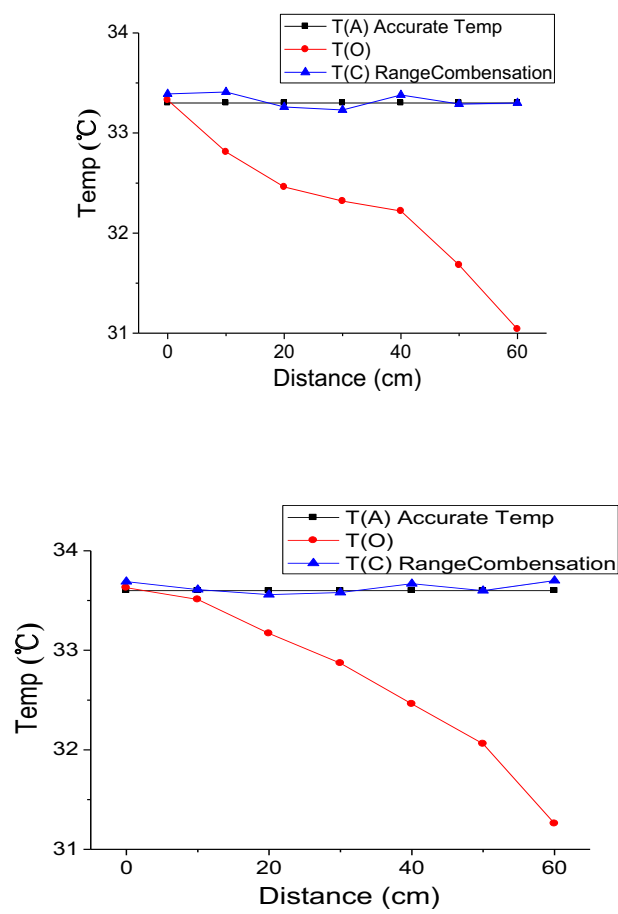

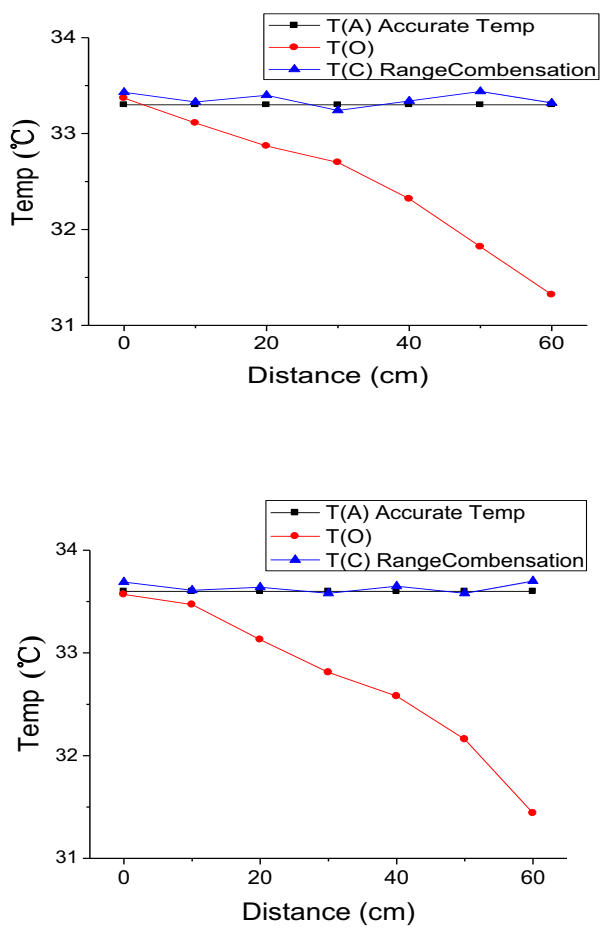

Figure 3. T (O), T (A) , T(C) of four subjects

As the fitting algorithm used the data from the 6 points from 0 to $60 \mathrm{~cm}$, in order to ensure the reliability of the system in the full range of 0 to $60 \mathrm{~cm}$, randomly selected subject A to measure temperature at random distances from 0 to $60 \mathrm{~cm}$, and the recorded results were shown in Figure 4.

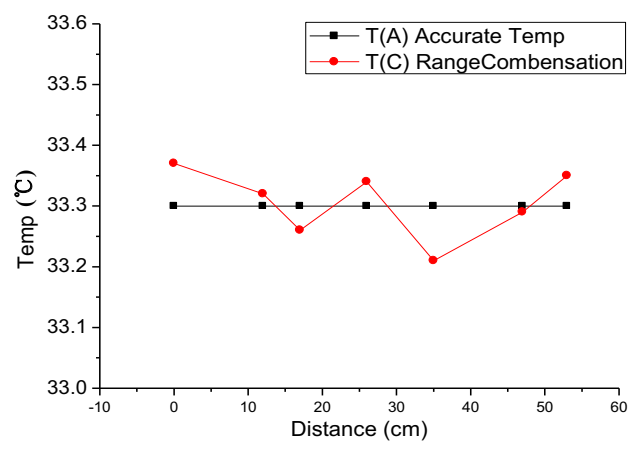

Figure 4. T(C), T (A) of subject A

In order to further explore the effect of skin color had on the accuracy of the system temperature measurement, two subjects were randomly coated with black and white edible pigments on the forehead to simulate the black and white complexion. The results of the two sets of tests measured by the infrared temperature measurement system with distance compensation in the range of 0 $60 \mathrm{~cm}$ were compared with the actual temperature values measured by Microlife FR1DM1, and the maximum error was no more than $0.2^{\circ} \mathrm{C}$. Figure 5 was the result of black skin and white skin. It could be seen that the skin color had no effect on the system measurement accuracy.
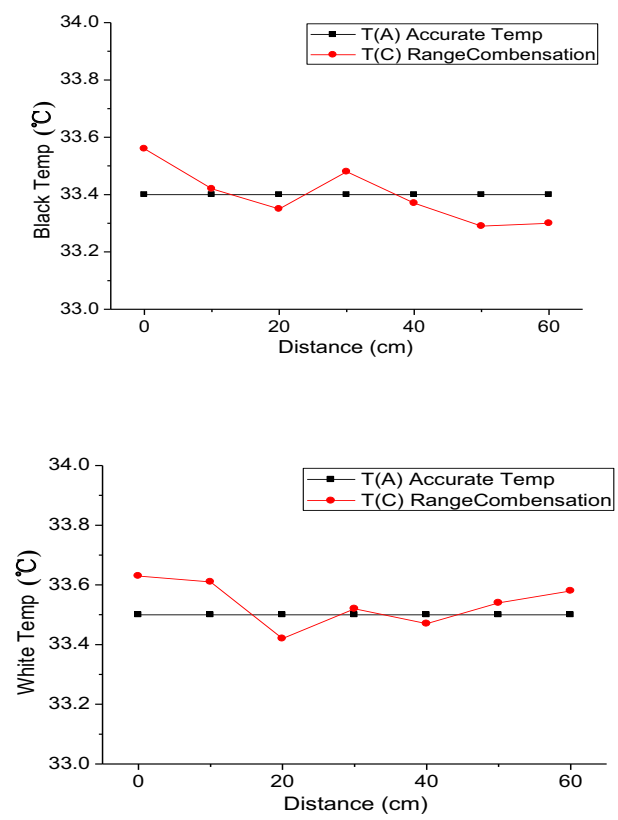

Figure 5. T(C), T (A) of black and white skin

\section{Results and Discussion}

The errors of the infrared temperature measurement system, based on distance compensation of four yellow skins, a simulated black skin, and a simulated white skin have been shown in table 2 , where $\mathrm{D}(\mathrm{cm})$ is the distance between the temperature measurement system and the irradiated part of the subject, $\varepsilon$ is the error between the forehead temperature $\mathrm{T}(\mathrm{C})$ measured by the infrared temperature measurement system based on distance compensation and the actual temperature value $\mathrm{T}(\mathrm{A})$ measured by Microlife FR1DM1, $\varepsilon \operatorname{MAX}\left({ }^{\circ} \mathrm{C}\right)$ is the maximum error between $\mathrm{T}(\mathrm{C})$ and $\mathrm{T}(\mathrm{A})$. It could be seen that the temperature measurement accuracy of the infrared temperature measurement system based on distance compensation has been improved, and the maximum measurement error in the range of $0-60 \mathrm{~cm}$ is kept within $0.2^{\circ} \mathrm{C}$ and the measurement result has not affected by the skin color.

Table 2. Error of forehead temperature

\begin{tabular}{|c|c|c|c|c|c|c|}
\hline \multirow{2}{*}{$\begin{array}{c}\text { NO. } \\
\text { D(cm) }\end{array}$} & $\mathrm{A}$ & $\mathrm{B}$ & $\mathrm{C}$ & $\mathrm{D}$ & BLACK & WHITE \\
\cline { 2 - 7 } & \multicolumn{7}{|c|}{$\varepsilon=\mathrm{T}(\mathrm{C})-\mathrm{T}(\mathrm{A}){ }^{\circ} \mathrm{C}$} \\
\hline 0 & 0.09 & 0.09 & 0.13 & 0.09 & 0.16 & 0.13 \\
\hline 10 & 0.11 & 0.01 & 0.03 & 0.01 & 0.02 & 0.11 \\
\hline 20 & -0.04 & -0.04 & 0.1 & 0.04 & -0.05 & -0.08 \\
\hline 30 & -0.07 & -0.02 & -0.06 & -0.02 & 0.08 & 0.02 \\
\hline 40 & 0.08 & 0.07 & 0.04 & 0.05 & -0.03 & -0.03 \\
\hline 50 & -0.01 & 0 & 0.14 & -0.02 & -0.11 & 0.04 \\
\hline 60 & 0 & 0.1 & 0.02 & 0.1 & -0.1 & 0.08 \\
\hline $\begin{array}{c}\varepsilon_{\mathrm{MAX}} \\
\left({ }^{\circ} \mathrm{C}\right)\end{array}$ & 0.11 & 0.1 & 0.14 & 0.1 & 0.16 & 0.13 \\
\hline
\end{tabular}


During the test, 20 sets of real time data have been collectedper second and the system response is very sensitive and the response time of the system is less than $0.1 \mathrm{~s}$.

It could be seen from the above results, the infrared temperature measurement system based on distance compensation, in the range of $0-60 \mathrm{~cm}$, the temperature measurement accuracy was $0.2^{\circ} \mathrm{C}$, the system response time was less than $0.1 \mathrm{~s}$. The system could meet the need of the optical rehabilitation therapy and other long-range non-contact measurement. However, due to the limited sample of the experiment, the fitting formula used in this system needs further verification. The follow-up work is to collect more diversified data and find a more precise temperature compensation formula.

\section{Conclusion}

To meet the need of real-time remote monitoring human body surface temperature for optical rehabilitation therapy, a non-contact high-precision real-time temperature measurement method based on distance compensation has been proposed, and the system design is carried out. In order to verify the accuracy of the system, six different subjects were tested. The results show that the designed non-contact infrared temperature measurement system has a residual error of less than $0.2^{\circ} \mathrm{C}$ and a response time of less than $0.1 \mathrm{~s}$ in the range of $0-60 \mathrm{~cm}$. This provides a reference for developing longdistance temperature measurement equipments in optical therapy.

\section{References}

1. Zhen Yang,Shi Cheng Zhang,Li Yang. Altering Spectrum Method in Temperature Measurement Using Infrared Imager in Complex Background Environment[J]. Advanced Materials Research (2012)

2. Wiezorrek R,Bialasiewicz A A,Engelmann K,Grasedyck K,Richard G. [Necrotizing keratitis in chronic polyarthritis. Combined immunosuppressive and surgical therapy].[J]. Der Ophthalmologe : Zeitschrift der Deutschen Ophthalmologischen Gesellschaft, 95, 9 (1998)

3. Westermann Simone,Buchner Heinz H F,Schramel Johannes P,Tichy Alexander,Stanek Christian. Effects of infrared camera angle and distance on measurement and reproducibility of thermographically determined temperatures of the distolateral aspects of the forelimbs in horses.[J]. American Veterinary Medical Association. Journal(2013)

4. Zhang $\mathrm{Y}$, Wang $\mathrm{X} \mathrm{S}$, Ming $\mathrm{H}$ E. Influence of Distance on Temperature Measurement Accuracy of Infrared Thermal Imager and Error Correction[J]. Infrared (2011)

5. Zhang Y C, Chen Y M, Fu X B, et al. A method for reducing the influence of measuring distance on infrared thermal imager temperature measurement accuracy [J]. Applied Thermal Engineering (2016) 